\title{
Simulation of energy consumption in urban areas.
}

\author{
Nikolay Garyaev * \\ Moscow State University of Civil Engineering; 26, Yaroslavskoe Shosse, Moscow, 129337, Russia
}

\begin{abstract}
One of the problems that may arise in the way of successful implementation of energy supply in urban areas is the difficulty of analyzing and interpreting a large amount of digital data received from various sensors. This problem may adversely affect the performance of energy organizations. The purpose of this study is to study modern tools to solve the problem of processing big data using technologies of simulation and artificial intelligence. This study is dedicated to the development of innovative digital models for the balanced distribution of energy consumption in urban areas.
\end{abstract}

\section{Introduction}

To solve the problem of lack of resources and a balanced distribution of energy consumption in urban areas, you can use the methods of simulation and artificial intelligence (AI). The current level of development of information technologies and intelligent systems based on them allows us to implement this task.

It is important to note that supporters of the implementation of simulation and $\mathrm{AI}$ in the electricity supply system are various environmental organizations. They insist on the need to increase the number of renewable energy facilities and reduce the number of traditional coal and gas power plants, as well as the use of energy-saving technologies.

Thus, there is an urgent need to develop an intelligent system for analyzing the demand for electricity in urban areas at different time periods. In addition, to ensure maximum accuracy in forecasting energy demand, such a system should take into account changes in weather conditions to form an indicator of electricity demand [1]. A large-scale project in this direction is being implemented by Google. The high-tech giant is working on the implementation of a system for analyzing the needs of the population for electricity in order to optimize the supply of electricity.

In Russia, for the purpose of monitoring and analyzing energy supply, devices are installed that are equipped with special software that allows you to remotely control the amount of electricity consumed in each house and apartment.

The accumulated significant amounts of energy consumption data, the so-called BIG DATA, can only be quickly analyzed using modern approaches to analysis and decision-making on managing a complex energy supply system.

The current approaches to solving problems based on machine intelligence make it possible to combine interdisciplinary knowledge and reveal hidden patterns in various areas of science [2-7].
Existing solutions today are difficult to call artificial intelligence, because only its individual fragments, technologies and methods are implemented.

So far, artificial intelligence has not been implemented to the necessary extent, since a computer comparable to the human brain has not been created. It is important to note that artificial intelligence is not only a supercomputer, but also software algorithms.

Implementation of the development of simulation models and AI of the required level, i.e. capable of solving creative problems, it will require decades of research by many thousands of laboratories and institutes; it may require a fundamentally different hardware base and new fundamental discoveries.

Since there is still no established definition for artificial intelligence, the following definition can be given - it is a computer system capable of learning, using accumulated knowledge and experience, solving creative and inventive problems, not using enumeration methods, building strategies and applying abstract concepts.

It's no secret that technological lag worsens the economic and political prospects of any state, so in the coming years all the leading countries will be embroiled in a race of innovative technologies. The introduction of AI technologies allows us to provide significant economic advantages due to the effective redistribution of energy and other resources and ensures security in the production and information spheres, as well as in the military-technical field.

This is confirmed by the words from the speech of President V.V. Putin at an open lesson in Yaroslavl on September 1, 2017: "Artificial intelligence is not only the future of Russia, it is the future of all mankind. Here are tremendous opportunities and threats that are difficult to predict today. Anyone who becomes a leader in this field will be the ruler of the world." 


\section{Tasks of simulation and analysis with elements of artificial intelligence in the field of energy supply in urban areas.}

In the foreseeable future, the global technology race will lead to the introduction of the most modern innovations in the energy supply of urban areas [8-10]. This applies not only to new areas of development, but also to the existing housing stock.

The primary tasks of simulation and AI in the field of energy supply in urban areas are:

1. Ensuring the operation of the energy supply system in optimal mode;

2. Effective management and reliable energy supply to consumers;

3. Effective use of technical capabilities of power equipment;

4. Monitoring the technical condition and troubleshooting of power equipment;

5. Localization of emergency and emergency situations;

6. Collection of data on events occurring in the power system;

7. Ensuring the rational use of fuel and energy resources and the management of energy consumption regimes;

8. Accounting for fuel and energy resources and forecast their needs;

9. Planning of technological inspections and repairs, control of operational parameters of power system elements.

\section{The concept of collecting and analysing big data (BIG DATA) for monitoring energy consumption in urban areas.}

The development of modern approaches to the design of engineering systems based on information modeling (information model of a building) increasingly leads to the need to equip them with sensors to collect information throughout the entire life cycle of an object. Sensor-based building information can be provided in real time, processed and linked to information from other sources to gain comprehensive knowledge of the respective systems (Industrie 4.0, Semantic Web, Internet of Things, "Intelligent Bridge"). The term "intelligent building" is understood to mean a system that provides security, resource saving and comfort, recognizing specific situations occurring in the building and correspondingly responding to them according to previously developed algorithms. The engineering structure and building monitoring system must be considered as a whole. However, in existing concepts of such structures, additional accounting information based on sensors is not used and is not taken into account. For example, the system of European standards Eurocodes and the basic concept of partial security are based on the assumption that information about the actual state of the construction site is not available throughout the entire life cycle of the building. Instead, the design process uses assumptions that take into account the effect of uncertainties on the entire life cycle of the building [11]. With this approach to the design of engineering systems and their subsequent operation, significant additional costs may arise, which subsequently often manifests itself in additional activities that cannot be optimally planned, but which affect the energy efficiency of the building [12].

The presented research project is aimed at developing the concept of collecting and analyzing big data and has the following objectives:

1. To study the Big Data Analysis (BIG DATA) approaches obtained from sensors for collecting information about engineering systems throughout the entire life cycle of an object, for accounting and analysis of energy consumption.

2. To develop the general concept of a monitoring system for intelligent buildings and engineering structures based on simulation models and AI.

The main objective of the study is to develop a methodology for the formation of basic data types for subsequent analysis, an example of the data in Figure 1. Formation of model classes for machine learning, descriptive analysis and optimization, development of models for MapReduce, Hadoop and NoSQL, taking into account the complex structural interaction of the entire engineering system.

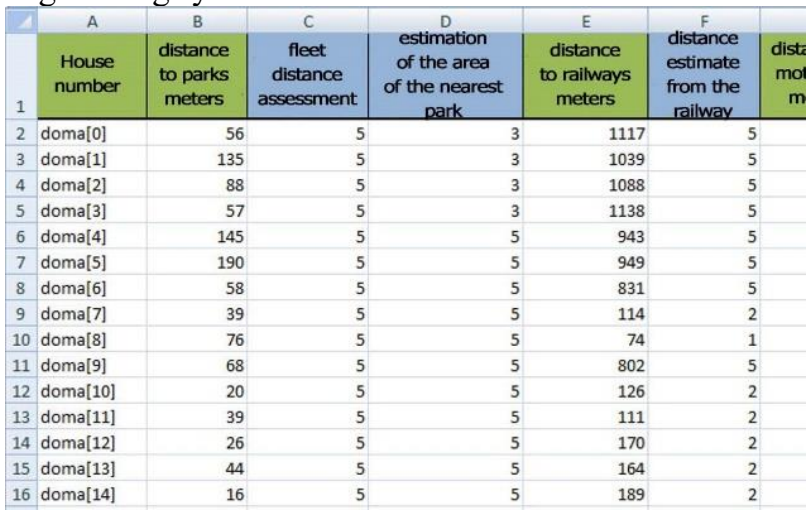

Fig. 1 Data fragment for all houses of a part of the city territory.

In addition, it is necessary to take into account the uncertainties that are caused by the system itself and the connected hardware and software systems (for example, sensor failure, sensor error calibration, interrupted data transfers). These additional uncertainties should be analyzed and integrated into the approach being developed in order to reduce the uncertainty of these impacts.

\section{Development of a simulation model of energy consumption in urban areas.}

Russian science has good prospects to participate in the global process of acquiring new knowledge in the exact sciences, such as mathematics, physics, chemistry, and to continue research in the field of modeling and AI. It is important to note that the work of scientists in this field 
is closed in nature and the results of research are not made publicly available.

As the research software, the AnyLogic simulation tool was used to create virtual simulation environments, including for testing artificial intelligence systems and machine learning.

The aim of this work is to develop a simulation model of energy consumption in urban areas using the example of a given area, with elements of artificial intelligence and machine learning. The simulation model is based on the analysis of cartographic data and the results of the collection and analysis of big data (BIG DATA) for monitoring the energy consumption of the study area. For each parameter, indicators were selected and used in accordance with their purpose. The possibility of changing the model according to given indicators when changing criteria was taken into account.

The simulation model provides a comprehensive assessment of the energy consumption of the area according to the selected parameters. The developed model has the following characteristics:

- accessibility and simplicity of the model (the user does not have to have programming skills to use the model or change the input parameters);

- selection of an environment that allows the use of external data sources;

- the final model is universal, (does not require special technical or paid software);

- the ability to change the values of indicators;

- the model takes into account the dynamic changes made by the user and displays them;

Figure 2 shows a screenshot of the results of the simulation model.

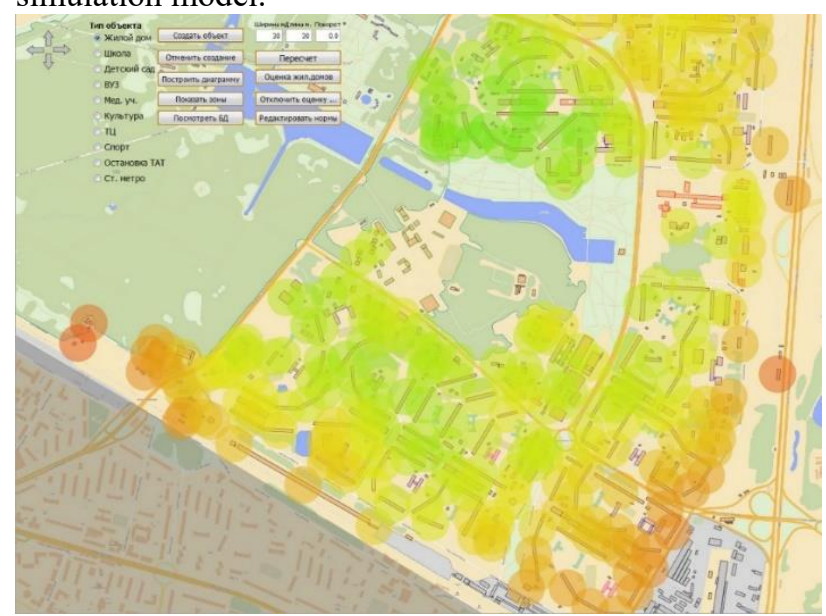

Fig. 2 Energy consumption of the studied part of the urban area.

The developed model allowed, in addition to solving the tasks set, to move on to solving the problem of sustainable development of cities and urban territories. For each district to be evaluated, individual assessment criteria are selected from the generated database, which are converted for clarity into a pie chart Figure 3.

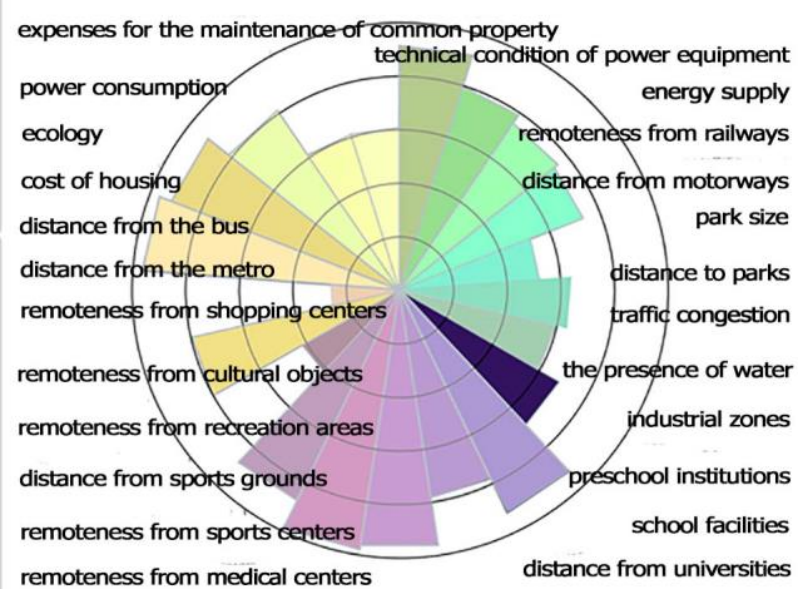

Fig. 3 Diagram of sustainable development of the urban area of the studied area.

Based on the results of computer modeling, qualitative conclusions have been obtained that make it possible to detect such properties of the system under study as the dynamics of its development, structure, integrity, stability, and others. Quantitative results allow us to characterize the system with the values of past variables and the forecast of future ones. The most important area of application of computer modeling is finding the highest performance indicators of the model as a result of the search for optimal options for external impact on the object.

\section{Conclusions}

The created model allows you to visually assess the urban area by energy consumption and display a graphical and textual interpretation of the required data in a convenient form for analysis. The use of AI elements and machine learning on real data allows us to obtain predicted energy consumption values of various urban areas, however, the results obtained require further research to form a full-scale model that takes into account many other influential factors in addition to energy consumption.

The model allows you to predict the change in the studied parameters over time, as well as compare and evaluate data for compliance with regulatory requirements. In addition, it is possible to simulate various situations at the discretion of the user, followed by a detailed analysis of the data for each object.

The most popular areas of application of the developed model, bringing the greatest effect, are the tasks:

- forecasting (condition of equipment, changes in consumption, etc.);

- optimization (operation parameters of power system elements, consumption, networks, etc.);

- control (lighting, energy sources and batteries, etc.);

- communications (energy supply companies with consumers).

In conclusion, it should be noted that the demand for the use of simulation tools and artificial intelligence in the energy sector is associated, firstly, with the energy 
transformation due to the increased use of renewable energy sources, the intellectualization of energy production, transmission and consumption (smart technology), and secondly with the digital transformation caused by the growing need for monitoring and analysis of data (big data) and the introduction of new technologies (for example, blockchain, "qi background substation ", unmanned devices, etc.).

\section{Thankfulness}

This study was performed with the financial support of the RF Ministry of Education and Science, President Grant \#NSh-3492.2018.8

\section{References}

1. Tiansen Liu, Dapeng Liang, Xinpeng Xing, Pei Kuang, Yufeng Zhang Does energy consumption endanger public longevity? An analysis of 34 major energy-consuming countries International Journal of Smart Grid and Clean Energy Vol. 8, No. 5, September 2019

2. Adamtsevich A., Ginzburg A., Shilova L., Shilov L. Implementation of BIM-technologies in Russian construction industry according to the international experience / Journal of Applied Engineering Science, 14 (4), p. 457-460 (2016).

3. Afanas'ev A., Ginzburg A., Kozhevnikova S., Stepanov V. Organization of concrete works on the bases of the information system of tracking / Proceedings of the 19th International Scientific Conference "Energy Management of Municipal Transportation Facilities and Transport EMMFT 2017”. Khabarovsk, Russia 10-13 April 2017.
Advances in Intelligent Systems and Computing, vol 692. Springer, Cham. https://doi.org/10.1007/978-3319-70987-1_126.

4. AI Now Report 2018. AI Now Institute, New York University. Dezember 2018.

5. Digitalisierung im Energiesektor. Dialogpapier zum Transformationsprozess. Bundesamt für Energie BFE. Bericht vom 11. Dezember 2018.

6. Garyaev N.A. Design and analysis of information model hotel complex. MATEC Web of Conferences 2016. C. 06007.

7. Real-Time Loss Prediction. T\&D World Magazine. November 2018

8. Garyaev N.A. Geographically distributed learning network construction industry. Computing in Civil and Building Engineering Proceedings 2014 International Conference. 2014. C. 1578-1585.

9. Garyaeva V.V. Application of BIM modeling for the organization of collective work on a construction project/ MATEC Web of Conferences 2018. C. 05025 .

10. Garyaeva V.V., Garyaev N.A. Integrated assessment of the technical condition of the housing projects on the basis of computer technology. Computing in Civil and Building Engineering Proceedings 2014 International Conference. 2014. C. 1336-1343.

11. Garyaev P.N. Computer-aided zoning and urban planning. Computing in Civil and Building Engineering Proceedings 2014 International Conference. 2014. C. 1618-1625.

12. World Intellectual Property Organization (WIPO) Technology Trends 2019. Artificial Intelligence. 2019. 